\title{
(6) OPEN ACCESS \\ Radiographic endophenotyping in hip osteoarthritis improves the precision of genetic association analysis
}

\author{
Kalliope Panoutsopoulou, ${ }^{1}$ Shankar Thiagarajah, ${ }^{2}$ Eleni Zengini, ${ }^{2,3}$ \\ Aaron G Day-Williams, ${ }^{1,4}$ Yolande FM Ramos, ${ }^{5}$ Jennifer MTA Meessen, ${ }^{5,6}$ \\ Kasper Huetink, ${ }^{6}$ Rob GHH Nelissen, ${ }^{6}$ Lorraine Southam ${ }_{1}^{1,7}$ N William Rayner, ${ }^{1,7,8}$ \\ arCOGEN Consortium, Michael Doherty, ${ }^{9}$ Ingrid Meulenbelt, ${ }^{5}$ Eleftheria Zeggini, ${ }^{1}$ \\ J Mark Wilkinson ${ }^{2}$
}

\begin{abstract}
Handling editor Tore K Kvien
- Additional material is published online only. To view please visit the journal online (http://dx.doi.org/10.1136/ annrheumdis-2016-210373)

For numbered affiliations see end of article.
\end{abstract}

\section{Correspondence to} Professor J Mark Wilkinson, Metabolic Bone Unit, Sorby Wing Northern General Hospital, Sheffield S5 7AU, UK; j.m.wilkinson@sheffield.ac.uk

$\mathrm{KP}, \mathrm{ST}, \mathrm{IM}, \mathrm{EZ}$ and JMW contributed equally.

EZ and JMW are joint corresponding authors.

Received 16 August 2016 Revised 14 November 2016 Accepted 23 November 2016 Published Online First 14 December 2016

\section{ABSTRACT}

Objective Osteoarthritis (OA) has a strong genetic component but the success of previous genome-wide association studies (GWAS) has been restricted due to insufficient sample sizes and phenotype heterogeneity. Our aim was to examine the effect of clinically relevant endophenotyping according to site of maximal joint space narrowing (maxJSN) and bone remodelling response on GWAS signal detection in hip OA.

Methods A stratified GWAS meta-analysis was conducted in 2118 radiographically defined hip OA cases and 6500 population-based controls. Signals were followed up by analysing differential expression of proximal genes for bone remodelling endophenotypes in 33 pairs of macroscopically intact and OA-affected cartilage.

Results We report suggestive evidence $\left(p<5 \times 10^{-6}\right)$ of association at 6 variants with $O A$ endophenotypes that would have been missed by using presence of hip OA as the disease end point. For example, in the analysis of hip OA cases with superior maxJSN versus cases with non-superior maxJSN we detected association with a variant in the $L R C H 1$ gene ( $r 5754106, p=1.49 \times 10^{-7}$, OR ( $95 \% \mathrm{Cls}) 0.70(0.61$ to 0.80$))$. In the comparison of hypertrophic with non-hypertrophic OA the most significant variant was located between STT3B and

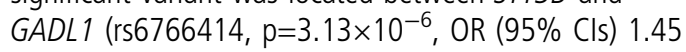
(1.24 to 1.69)). Both of these associations were fully attenuated in non-stratified analyses of all hip OA cases versus population controls ( $p>0.05$ ). STT3B was significantly upregulated in $\mathrm{OA}$-affected versus intact cartilage, particularly in the analysis of hypertrophic and normotrophic compared with atrophic bone remodelling pattern $\left(p=4.2 \times 10^{-4}\right)$.

Conclusions Our findings demonstrate that stratification of OA cases into more homogeneous endophenotypes can identify genes of potential functional importance otherwise obscured by disease heterogeneity.

\section{CrossMark}

To cite: Panoutsopoulou $K_{\text {, }}$ Thiagarajah S, Zengini $E$, et al. Ann Rheum Dis 2017;76:1199-1206.

\section{INTRODUCTION}

Osteoarthritis (OA) is a phenotypically heterogeneous disease characterised by cartilage degeneration and bone remodelling within synovial joints, ${ }^{1}$ and affects 9 million people in the UK alone. ${ }^{2}$ The heritable component to $\mathrm{OA}$ susceptibility is estimated at $40 \%-65 \%,{ }^{3-5}$ however fewer than 20 robustly associated genetic loci have been identified to date explaining $<10 \%$ of this heritability. ${ }^{6-18}$ These candidate gene and genome-wide association studies (GWAS) used a simple dichotomous description of joint involvement that does not reflect the heterogeneity of OA biology, ${ }^{19}$ with the exception of the DOT1L association with hip OA in men that was made by measurement of cartilage thickness. ${ }^{20}$

OA endophenotypes may be characterised on plain radiographs by the pattern of joint involvement and by the bone remodelling response to the disease. ${ }^{21-23}$ At the hip, the site of maximum joint space narrowing (maxJSN) can be classified as superior, medial, axial or concentric. ${ }^{22} 2425$ The superior pattern is most common in men and is a risk factor for disease progression. ${ }^{26} 27$ Medial migration is more common in women and more likely to occur bilaterally. ${ }^{26-30}$ The bone remodelling response to $\mathrm{OA}$ also varies between individuals, and may be classified as atrophic (bony attrition, cysts and a lack of osteophytes), normotrophic (osteophytes <grade 3) or hypertrophic (osteophytes $\geq$ grade $3 \pm$ increased femoral head size). ${ }^{23}$ Atrophic OA is associated with thinner trabeculae, lower bone volume and lower bone mineral density than the hypertrophic or normotrophic patterns of OA. ${ }^{31} 32$ Atrophic OA is also associated with faster disease progression, osteoporosis and with femoral head collapse. ${ }^{28} 33$ The risk of severe hip OA is threefold greater in siblings of index cases with an atrophic pattern of hip OA compared with siblings whose index case had a normotrophic or hypertrophic bone remodelling response. $^{27}$

Here, we applied these clinically relevant radiographic endophenotypes to identify novel variants that associate with hip OA in a subset of subjects from the arcOGEN Study, ${ }^{14}$ the largest hip and knee OA GWAS to date. We calculated the differences in statistical power achieved using this approach versus a simple dichotomous description of joint involvement. We investigated the effect of using these endophenotype definitions on signal detection for previously established hip OA loci. Finally, we examined the expression levels of genes highlighted in the association analysis in primary 
human chondrocytes taken from patients classified using the same bone remodelling endophenotypes.

\section{PATIENTS AND METHODS}

\section{Study populations and radiographic classification}

The hip OA subjects comprised 2118 unrelated individuals of UK European ancestry participating in the arcOGEN Study, an ethically approved study in which all subjects provided written, informed consent prior to participation. ${ }^{14}$ All had radiographic evidence of hip OA (Kellgren-Lawrence (KL) Score $\geq 2$ ) on a digitised anteroposterior radiograph of the affected joint. ${ }^{21} \mathrm{Hip}$ OA pattern was classified by the site of maxJSN as superior, axial, medial or concentric using the Osteoarthritis Research Society International (OARSI) atlas and the KL Score (table 1). ${ }^{21} 22$ The bone remodelling response was classified according to the Bombelli and OARSI atlases as atrophic, normotrophic or hypertrophic. ${ }^{22} 23$ Each radiograph was read independently by two clinically trained observers (ST and EZen). Where discrepancy existed between the observers, adjudication was made by the senior clinical author (JMW). The correlation between the two primary observers was 'very good' for the axial and medial JSN phenotypes $(\kappa=0.85,95 \% \text { CI } 0.82 \text { to } 0.88)^{34}$ and 'good' for the superior JSN phenotype $(\kappa=0.7,95 \%$ CI 0.67 to 0.73$)$; 'good' for the atrophic remodelling phenotype $(\kappa=0.64,95 \%$ CI 0.58 to 0.69 ) and 'moderate' for the hypertrophic phenotype $(\kappa=0.54,95 \%$ CI 0.50 to 0.58$)$.

\section{Genotyping, imputation and association analyses}

Genotypes were extracted from 1817 and 301 hip OA cases previously genotyped on Human 610-Quad and HumanOmniExpress (Illumina, San Diego, USA) platforms, respectively (figure 1). The controls comprised 6500 subjects previously included in the arcOGEN GWAS: The Wellcome Trust Case Control Consortium 2 Study 1958 Birth Cohort, the UK National Blood Donor Service and the Type 1 Diabetes Genetics Consortium. ${ }^{14}$ A 1000 Genomes Project-based imputation was conducted, ${ }^{35} 36$ and was followed by stringent quality control excluding variants with minor allele frequency $(\mathrm{MAF})<0.01$ and minor allele count $<10$ given the different sample sizes of the GWAS case cohorts, ${ }^{37}$ imputation information score $<0.4$ and by inspecting genotype intensity plots $100 \mathrm{~kb}$ on either side of the top signals and re-imputation of these regions of association.

Association analyses at $\sim 7$ million variants were made under an additive model (maximum likelihood ratio test, SNPTESTv2.5). Our primary analyses comprised fixed-effect meta-analyses ${ }^{38}$ within OA cases stratified by site of maxJSN or by bone remodelling response to identify variants exclusively associated with these endophenotypes (table 1 and figure 1). The axial and medial maxJSN groups were collapsed into one (axial/medial) due to the small sample size in each of these categories. Promising signals were independently validated by de novo genotyping using the MassARRAY iPLEX Gold assay (Agena Bioscience GmbH, Hamburg, Germany).

In our secondary analyses the GWAS outputs for the stratified hip OA cases against population-based controls were compared with that achieved using a non-stratified GWAS of all hip OA cases versus population-based controls. Power was calculated using Quanto V.1.2.4 under the additive model ${ }^{39}$ at $\alpha=5 \times 10^{-8}$, and based on study-specific effect sizes and case-control ratios identified in the Human 610-Quad GWAS. Similarly, the sample size required to have $80 \%$ power to detect the associated signals with genome-wide significance $\left(\mathrm{p}=5 \times 10^{-8}\right)$ was estimated. To calculate the sample size fold reduction afforded by the use of precise phenotype definitions over an all hip OA versus controls approach we fixed the estimated sample size of hip OA cases from infinite to $n=1000000$.

\section{Gene expression analysis}

Gene expression profiles were examined in samples from the RAAK Study (Research Arthritis and Articular Cartilage) from individuals undergoing joint replacement surgery, as described previously. ${ }^{40}$ Isolated RNA was prepared for hybridisation using the Illumina TotalPrep RNA Amplification Kit (Illumina, San Diego, USA) onto Illumina HumanHT-12 v3 microarrays, with each pair (OA-affected and intact) measured on the same chip. Microarrays were read using an Illumina Beadarray 500GX scanner and data were analysed in R statistical programming language after quality checks using Illumina Beadstudio analysis software (Illumina). Intensity values were normalised and absence of large-scale between-chip effects was confirmed using the Globaltest-package. ${ }^{41}$ Probes that were not optimally measured were removed (detection $\mathrm{p}>0.05$ in more than $50 \%$ of the samples). All comparisons of the differential normalised probe expression levels between intact and diseased OA chondrocytes were made in SPSS (V.23, IBM, New York, USA) using the generalised estimation equations and corrected for age and sex. A random effect for patient identity (ID) was included to account for the structure of the data and correct for putative correlations between intact and OA-affected articular cartilage from the same joint.

Preoperative plain radiographs of the affected joint were scored independently by two experienced readers (JMTAM, $\mathrm{KH})$ to determine the OA subtype. Subsequently, osteophytes and JSN were graded 0-3 for lateral and medial femur and tibia (knee joints) and for inferior and superior femur and acetabulum (hip joints) by consensus opinion using the OARSI atlas. ${ }^{22}$

\section{RESULTS}

\section{Site of maxJSN association analysis}

In the GWAS meta-analysis of hip OA cases with superior maxJSN versus all other hip OA cases (see online supplementary figure $\mathrm{S} 1 \mathrm{a}$ ), the most significant association was observed at rs754106 $(\mathrm{MAF}=0.46)$ in intron 1 of the LRCH1 (leucine-rich repeats and calponin homology domain containing 1) gene $(\mathrm{T}$ allele $\mathrm{OR} \quad(95 \%$ CIs $) \quad 0.70 \quad(0.61$ to 0.80$)$, $\mathrm{p}=1.49 \times 10^{-7}$ (table 2 and see online supplementary figure S2a), and was consistent across both cohorts (see online supplementary table $\mathrm{S} 1$ ). In the GWAS meta-analysis of hip OA cases with axial/medial maxJSN versus all other hip OA cases (see online supplementary figure S1b), rs754106 was also the most significantly associated variant $\left(\mathrm{p}=3.66 \times 10^{-7}\right)$ but with the effect of the $\mathrm{T}$ allele in the opposite direction, in keeping with expectation (OR (95\% CIs) 1.47 (1.27 to 1.70), table 2 and see online supplementary table $\mathrm{S} 1$ ).

In the association analyses of hip OA cases with superior maxJSN versus population-based controls (see online supplementary figure S3a) and of patients with hip OA with axial/medial maxJSN versus population-based controls (see online supplementary figure S3b), opposite directions of effect were also observed for allele T: OR (95\% CIs) 0.91 (0.84 to 0.99 ), $\mathrm{p}=0.034$ compared with OR (95\% CIs) 1.32 (1.14 to 1.52 ), $\mathrm{p}=1.39 \times 10^{-4}$, respectively (see online supplementary table S1). When all hip OA cases were analysed together versus the same population-based control set, the signal was fully attenuated (OR (95\% CIs) 1.01 (0.93 to 1.08), p=0.89) (see online supplementary table S1 and figure S3e).

The second most significant finding in the superior maxJSN stratum versus all other hip $\mathrm{OA}$ cases was observed at 
Table 1 Patient characteristics

\begin{tabular}{|c|c|c|c|c|}
\hline OA phenotype & $\begin{array}{l}\text { arcOGEN_Illum610K } \\
\mathrm{N} \text { (men/women) }\end{array}$ & $\begin{array}{l}\text { arcOGEN_OmniExpress } \\
\mathrm{N} \text { (men/women) }\end{array}$ & $\begin{array}{l}\mathrm{N} \text { total } \\
\text { (men/women) }\end{array}$ & $\begin{array}{l}\text { Prevalence } \\
(\%)\end{array}$ \\
\hline Hip (KL Score $\geq 2$ ) & $1817(776 / 1041)$ & $301(125 / 176)$ & $2118(901 / 1217)$ & 100 \\
\hline \multicolumn{5}{|c|}{ Site of maximal joint space narrowing (pattern of femoral head migration within the acetabulum) } \\
\hline Axial & $158(34 / 124)$ & $28(5 / 23)$ & $186(39 / 147)$ & 8.8 \\
\hline Medial & $267(69 / 198)$ & $48(10 / 38)$ & $315(79 / 236)$ & 14.9 \\
\hline Superior & $1265(615 / 650)$ & $204(98 / 106)$ & $1469(713 / 756)$ & 69.3 \\
\hline Concentric & $127(58 / 69)$ & $21(12 / 9)$ & $148(70 / 78)$ & 7.0 \\
\hline \multicolumn{5}{|c|}{ Bone remodelling response } \\
\hline Atrophic & $267(113 / 154)$ & $18^{*}(8 / 10)$ & $285(121 / 164)$ & 13.4 \\
\hline Hypertrophic & $531(239 / 292)$ & $106(46 / 60)$ & $637(285 / 352)$ & 30.1 \\
\hline Normotrophic & $1019(424 / 595)$ & $177(71 / 106)$ & 1196(495/701) & 56.5 \\
\hline
\end{tabular}

*These individuals were not used as cases in the within-OA genome-wide association analysis of the arcOGEN OmniExpress data set due to the small sample number. $\mathrm{KL}$, Kellgren-Lawrence; OA, osteoarthritis.

A Primary analyses (within hip OA cases)

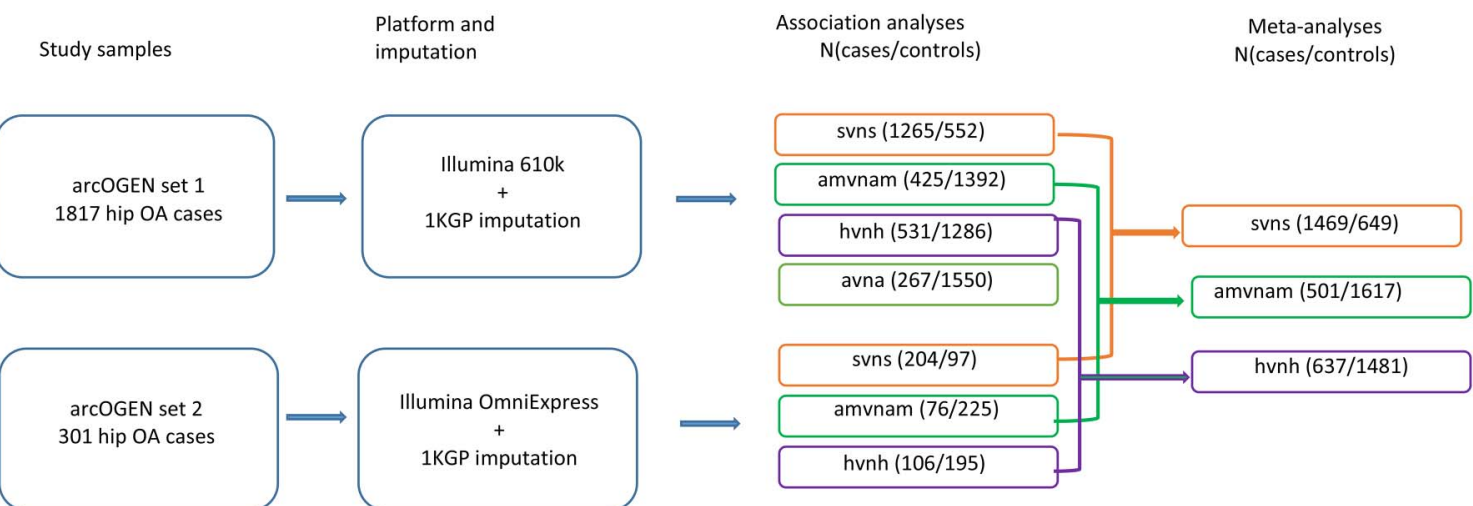

B Secondary analyses (hip OA cases vs. population-based controls)

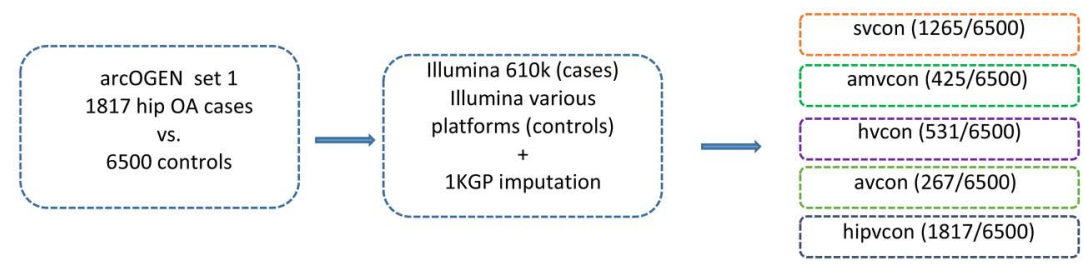

Figure 1 Genome-wide association study design, showing primary and secondary analyses. svns, superior joint space narrowing versus non-superior; amvnam, axial/medial joint space narrowing versus non-axial/medial; hvnh, hypertrophic bone response versus non-hypertrophic; avna, atrophic bone response versus non-atrophic; svcon, superior joint space narrowing versus population-based controls; amvcon, axial/medial joint space narrowing versus population-based controls; hvcon, hypertrophic bone response versus population-based controls; avcon, atrophic bone response versus population-based controls; hipvcon, hip osteoarthritis (OA) versus population-based controls.

rs73023563 (MAF $=0.19) \quad$ located between CWC22 (spliceosome-associated protein homologue (S. Cerevisiae)) and UBE2E3 (ubiquitin-conjugating enzyme E2E 3) (T allele OR (95\% CIs) 1.59 (1.33 to 1.91$), \mathrm{p}=6.59 \times 10^{-7}$, table 2 and see online supplementary figure S2b). The rs73023563 association was also strong in the converse stratum (hip OA cases with axial/medial maxJSN vs all other hip OA cases) with the opposite direction of effect ( $\mathrm{T}$ allele OR (95\% CIs) $0.61(0.50$ to $\left.0.75), p=2.03 \times 10^{-6}\right)$. Its association in the analysis of axial/ medial maxJSN cases versus population-based controls was equally strong (OR (95\% CIs) $0.63 \quad(0.52$ to 0.78$)$, $\left.\mathrm{p}=3.45 \times 10^{-6}\right)$, and was also fully attenuated in the all hip OA analysis versus population-based controls analysis (OR (95\% CIs) 0.94 (0.86 to 1.03$), \mathrm{p}=0.21$ ) (see online supplementary table $\mathrm{S} 1)$.
In the association analysis of hip OA with axial/medial maxJSN versus all other hip OA cases the second most significant single nucleotide polymorphism (SNP) was rs17050727 (MAF $=0.14)$, located between PRDM5 (PR domain containing 5) and MAD2L1 (mitotic arrest deficient-like 1 (yeast), T allele OR (95\% CIs) 1.65 (1.35 to 2.01 ), $\mathrm{p}=6.87 \times 10^{-7}$ (table 2 , see online supplementary table S1 and figure S2c). rs17050727 exhibited a similar pattern of association with the converse stratum (superior maxJSN vs all other hip OA cases) as for the variants described above; and its association was also fully attenuated in the hip OA analysis versus population-based controls analysis (OR (95\% CIs) 1.00 (0.90 to 1.12 ), $\mathrm{p}=0.98$, see online supplementary table $\mathrm{S} 1$ ).

Adjustments for sex, height and body mass index (BMI) had little effect on the association strength of the reported signals 
(see online supplementary table S2). Following validation experiments by de novo genotyping we found $100 \%$ genotype and minor allele concordance with GWAS genotypes of overlapping samples at rs754106 (see online supplementary table S3).

\section{Bone remodelling response association analysis}

In the GWAS meta-analysis of hypertrophic versus no-hypertrophic hip OA cases (see online supplementary figure S1c) the most significant SNP was rs6766414 (MAF=0.24) located between STT3B (subunit of the oligosaccharyltransferase complex (catalytic)) and GADL1 (glutamate decarboxylase-like 1), G allele OR (95\% CIs) 1.45 (1.24 to 1.69 ), $p=3.13 \times 10^{-6}$ (table 1 and see online supplementary figure S2d). We also found evidence of association in the hypertrophic OA versus population-based controls analysis, G allele OR (95\% CIs) 1.25 (1.09 to 1.44 ), $\mathrm{p}=1.74 \times 10^{-3}$ (see online supplementary table S1 and figure S3c). In the analysis of hip OA cases versus population-based controls the signal was fully attenuated (OR $(95 \%$ CIs) $1.00 \quad(0.92$ to 1.09$), \quad p=0.98) \quad$ (see online supplementary table $\mathrm{S} 1$ ).

The second most significant replicating SNP in the hypertrophic versus non-hypertrophic stratum, rs61837881 $(\mathrm{MAF}=0.24)$ located between C1orf65 and CAPN8 (calpain 8), was associated exclusively with the hypertrophic response (allele C: OR (95\% CIs) 1.44 (1.23 to 1.68$), p=4.56 \times 10^{-6}$, see online supplementary figure S2e) and was fully attenuated in the analysis of hip OA versus population-based controls (OR $(95 \%$ CIs) 1.06 (0.97 to 1.15$), p=0.23$, table 2 and see online supplementary table S1). Adjustment for sex, height and BMI had little effect on the association strength of the reported variants (table 2 and see online supplementary table S2).

In the GWAS of atrophic versus non-atrophic OA (see online supplementary figure S1d), the most significant association was observed at rs16869403, which resides within the gene encoding the $\mathrm{G}$ protein-coupled receptor 98 (GPR98) $(\mathrm{MAF}=0.10$, allele G OR (95\% CI) 2.11 (1.61 to 2.75$), p=1.37 \times 10^{-7}$, see online supplementary figure S2f and table S1). The strength of association was similar in the analysis of atrophic OA cases versus population-based controls (OR $(95 \% \mathrm{CI}) 1.93$ (1.52 to 2.46), $\mathrm{p}=4.14 \times 10^{-7}$, see online supplementary table $\mathrm{S} 1$ and figure S3d). This association was fully attenuated in the nonstratified hip OA versus population-based controls analysis (OR $(95 \% \quad \mathrm{CI}) \quad 1.05 \quad(0.93$ to 1.19$), \mathrm{p}=0.43$; see online supplementary table S1). Genotype and minor allele concordance between typed and imputed genotypes at this SNP were $99 \%$ and $97 \%$, respectively (see online supplementary table S3).

\section{Power gains}

Power increases substantially in the within-OA analyses compared with the non-stratified hip OA versus population-based controls analysis (table 3). Small to modest power increases are observed in the stratified OA cases versus population-based controls analyses compared with the non-stratified hip OA versus population-based controls analysis. The decrease in sample size required to reach genome-wide significance for an analysis within OA cases over the non-stratified hip $\mathrm{OA}$ versus population-based controls analysis ranged from 41-fold to 286 -fold. The decrease in sample size between stratified hip OA versus population-based controls analyses over the non-stratified hip OA versus population-based controls analysis ranged from 1 -fold to $>240$-fold. 
Table 3 Power gains associated with dichotomous versus specific radiographic endophenotype descriptors

\begin{tabular}{|c|c|c|c|c|c|c|c|c|c|c|}
\hline \multicolumn{7}{|c|}{ Study parameters } & \multicolumn{4}{|c|}{ Estimated parameters } \\
\hline SNP & Definition of cases & Definition of controls & $\begin{array}{l}\mathrm{N} \\
\text { cases }\end{array}$ & $\begin{array}{l}\mathrm{N} \\
\text { controls }\end{array}$ & EAF & OR & $\begin{array}{l}\text { Power } \\
(\%)\end{array}$ & $\mathrm{N}$ cases & $\mathrm{N}$ controls & $\mathrm{N}$ total \\
\hline \multirow[t]{3}{*}{ rs754106 } & Hip OA with superior JSN & Hip OA with non-superior JSN & 1265 & 552 & 0.54 & 0.72 & 18.45 & 2416 & 1054 & 3470 \\
\hline & Hip OA with superior JSN & Population-based & 1265 & 6500 & 0.54 & 0.91 & 0.05 & 10669 & 54821 & 65490 \\
\hline & Hip OA & Population-based & 1817 & 6500 & 0.54 & 1.01 & 0.00 & $>1000000$ & >3577325 & >4 577325 \\
\hline \multirow[t]{3}{*}{ rs73023563 } & Hip OA with superior JSN & Hip OA with non-superior JSN & 1265 & 552 & 0.19 & 1.63 & 57.78 & 1571 & 686 & 2257 \\
\hline & Hip OA with superior JSN & Population-based & 1265 & 6500 & 0.20 & 1.07 & 0.00 & 31513 & 161925 & 193438 \\
\hline & Hip OA & Population-based & 1817 & 6500 & 0.19 & 0.94 & 0.00 & 43995 & 157384 & 201379 \\
\hline \multirow[t]{3}{*}{ rs17050727 } & $\begin{array}{l}\text { Hip OA with axial/medial } \\
\text { JSN }\end{array}$ & $\begin{array}{l}\text { Hip OA with non-axial/medial } \\
\text { JSN }\end{array}$ & 425 & 1392 & 0.14 & 1.65 & 28.62 & 705 & 2309 & 3014 \\
\hline & $\begin{array}{l}\text { Hip OA with axial/medial } \\
\text { JSN }\end{array}$ & Population-based & 425 & 6500 & 0.14 & 1.43 & 4.89 & 1168 & 17864 & 19032 \\
\hline & Hip OA & Population-based & 1817 & 6500 & 0.14 & 1.00 & 0.00 & $\infty$ & $\infty$ & $\infty$ \\
\hline \multirow[t]{3}{*}{ rs6766414 } & $\begin{array}{l}\text { Hip OA with hypertrophic } \\
\text { response }\end{array}$ & $\begin{array}{l}\text { Hip OA with non-hypertrophic } \\
\text { response }\end{array}$ & 531 & 1286 & 0.24 & 1.42 & 12.40 & 1139 & 2758 & 3897 \\
\hline & $\begin{array}{l}\text { Hip OA with hypertrophic } \\
\text { response }\end{array}$ & Population-based & 531 & 6500 & 0.24 & 1.25 & 0.92 & 2199 & 26918 & 29117 \\
\hline & Hip OA & Population-based & 1817 & 6500 & 0.24 & 1.00 & 0.00 & $\infty$ & $\infty$ & $\infty$ \\
\hline \multirow[t]{3}{*}{ rs61837881 } & $\begin{array}{l}\text { Hip OA with hypertrophic } \\
\text { response }\end{array}$ & $\begin{array}{l}\text { Hip OA with non-hypertrophic } \\
\text { response }\end{array}$ & 531 & 1286 & 0.24 & 1.39 & 7.69 & 1298 & 3144 & 4442 \\
\hline & $\begin{array}{l}\text { Hip OA with hypertrophic } \\
\text { response }\end{array}$ & Population-based & 531 & 6500 & 0.24 & 1.33 & 7.15 & 1323 & 16195 & 17518 \\
\hline & Hip OA & Population-based & 1817 & 6500 & 0.24 & 1.06 & 0.00 & 40187 & 143762 & 183949 \\
\hline \multirow[t]{3}{*}{ rs16869403 } & $\begin{array}{l}\text { Hip OA with atrophic } \\
\text { response }\end{array}$ & $\begin{array}{l}\text { Hip OA with non-atrophic } \\
\text { response }\end{array}$ & 267 & 1550 & 0.10 & 2.11 & 58.36 & 330 & 1916 & 2246 \\
\hline & $\begin{array}{l}\text { Hip OA with atrophic } \\
\text { response }\end{array}$ & Population-based & 267 & 6500 & 0.10 & 1.93 & 42.25 & 383 & 9324 & 9707 \\
\hline & Hip OA & Population-based & 1817 & 6500 & 0.10 & 1.05 & 0.00 & 115540 & 413324 & 528864 \\
\hline
\end{tabular}

For each variant and stratum we present power (\%) based on study-specific effect size, effect allele frequency and case/control ratio from the GWAS on Illumina610k. Similarly we estimated the sample size required to have $80 \%$ power to detect the associated signals with genome-wide significance $\left(p=5 \times 10^{-8}\right)$.

EAF, effect allele frequency; GWAS, genome-wide association studies; JSN, joint space narrowing; OA, osteoarthritis; SNP, single nucleotide polymorphism.

\section{Established hip OA loci}

The strength of association of the 10 established hip OA susceptibility loci in Europeans ${ }^{18}$ was examined in each of the hip OA strata (see online supplementary table S4). Here, the risk allele of rs12982744 in DOTL1 associated with hip OA $\left(\mathrm{p}=3.06 \times 10^{-4}\right.$, OR (95\% CIs) 1.15 (1.07 to 1.24$\left.)\right)$ but the most significant association was observed in the analysis of hip OA with superior maxJSN versus population-based controls $\left(\mathrm{p}=8.17 \times 10^{-6}\right.$, OR $(95 \% \mathrm{CIs}) 1.22(1.12$ to 1.34$\left.)\right)$ (see online supplementary table S4). Further corroboration of this stratumspecific association comes from the within-OA GWAS analyses of the hip OA cases with superior maxJSN versus all other hip OA cases $\left(p=7.58 \times 10^{-3}\right.$, OR (95\% CIs) 1.22 (1.06 to 1.42$)$; and converse stratum $\mathrm{p}=1.13 \times 10^{-2}$, OR (95\% CIs) $0.81(0.69$ to 0.95$)$ ). The effect size differed between men and women both in our hip OA versus population-based control analysis (men $\mathrm{p}=8.92 \times 10^{-6}$, OR $(95 \%$ CIs) 1.30 (1.16 to 1.46$)$; women $\mathrm{p}=0.32$, OR (95\% CIs) 1.05 (0.95 to 1.17$)$ ), and in the superior maxJSN versus population-based controls analysis (men $\mathrm{p}=5.20 \times 10^{-6}$, OR (95\% CIs) 1.35 (1.18 to 1.53$)$; women $\mathrm{p}=0.07$, OR (95\% CIs) 1.12 (0.99 to 1.27$)$ ). This suggests that the sex disparity of effects at the DOT1L locus is not driven by the differences in prevalence of different JSN patterns between the two sexes.

Five other index SNPs (rs9350591, rs10948172, rs11177, rs4836732, rs6094710) also exhibited similar or lower $\mathrm{p}$ values and higher ORs in endophenotype-based association analyses than in the hip OA versus population-based controls analysis, despite the decrease in case sample size (ranging from 30\% to $77 \%$ ) (see online supplementary table S4). However, unlike the DOT1L variant, none of these showed an association that was strengthened enough to compensate for the multiple comparisons performed.

\section{Gene expression analyses in articular cartilage}

The expression levels of 10 genes located near variants highlighted in the bone remodelling stratified GWAS (GRP98 and ADGVR1 for rs16869403; STT3B, GADL1, OSBPL10, THRAP3 and TGFBR2 for rs6766414; and C1orf65, CAPN8 and SUSD4 for rs61837881) were examined in macroscopically intact and OA-affected articular cartilage $(n=33$ pairs from the RAAK Study, 6 with an atrophic, 19 with a normotrophic and 8 with a hypertrophic phenotype). We observed robust expression for SUSD4, STT3B, OSBPL10 and TGFBR2, with mean normalised probe level values of $7.9,7.8,8.4$ and 11.5 , respectively. Of these genes, only $S T T 3 B$ showed differential expression with respect to the disease process, with significant upregulation in OA-affected versus intact cartilage independent of joint site, particularly in patients with hypertrophic/normotrophic OA (fold change $=1.30, p=4.23 \times 10^{-4}$; figure 2 ). When the genotypes of rs6766414 (TT and TG) were tested, a significantly lower expression of $S T T 3 B$ in lesioned OA cartilage was observed among carriers of allele $G(n=9$ heterozygotes-no homozygotes for the $G$ allele were present in our data) compared with 


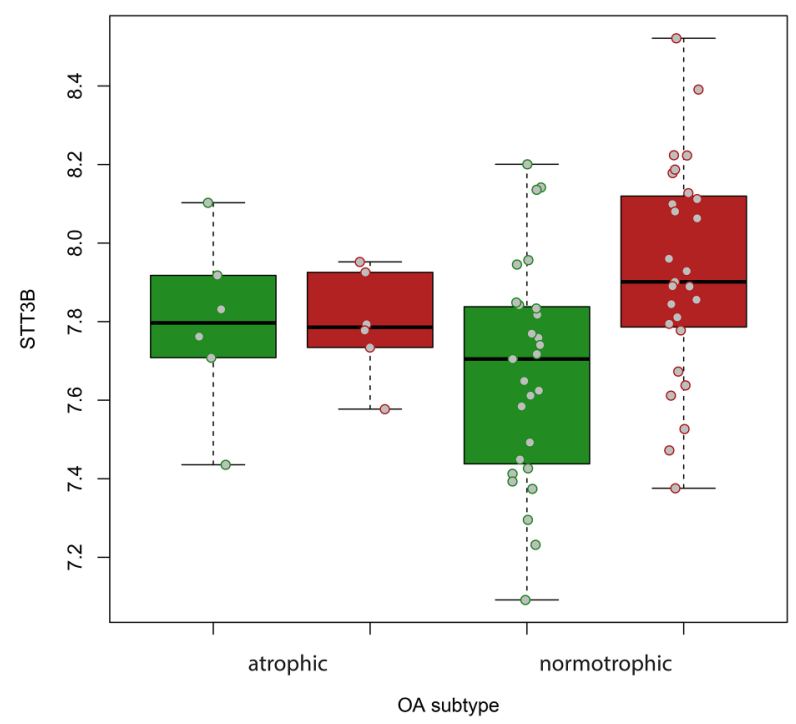

Figure 2 Box plots of the expression levels of STT3B plotted as the mean normalised probe level values in macroscopically intact (green boxes) and diseased (red boxes) osteoarthritis (OA) articular cartilage of patients with atrophic $(n=6)$ and normotrophic $(n=27)$ OA. Grey dots show values of each individual sample.

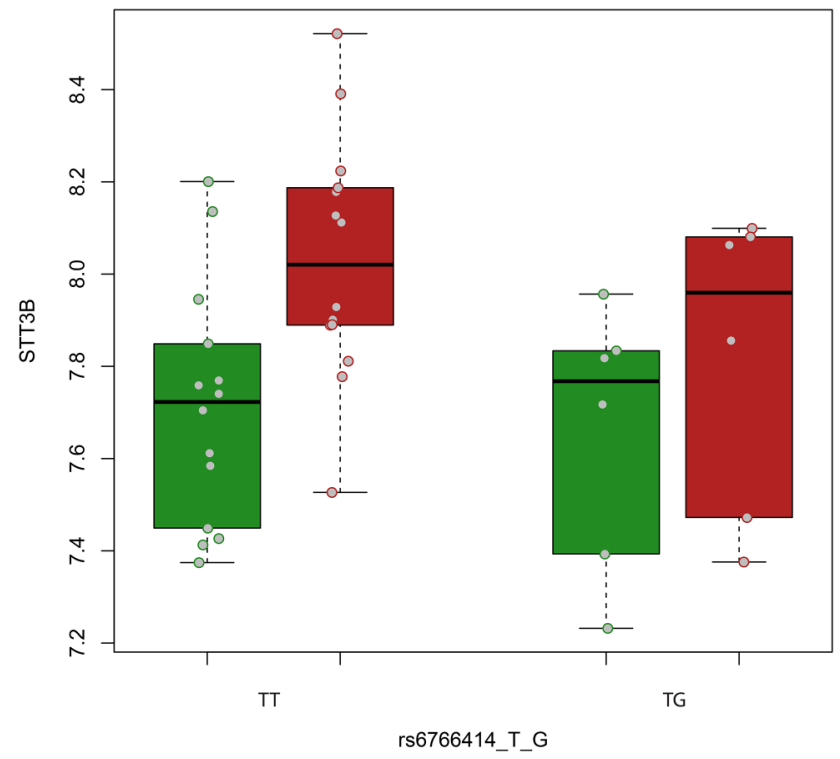

Figure 3 Box plots of the expression levels of STT3B in patients with hypertrophic/normotrophic osteoarthritis (OA), plotted as mean normalised probe level values, in macroscopically intact (green boxes) and diseased (red boxes) OA articular cartilage stratified by carriers of the $G$ allele (heterozygous individuals plotted since no homozygotes for the $G$ allele were present in our data) and by non-carriers at rs6766414. Grey dots show values of each individual sample.

homozygous individuals for allele $\mathrm{T}(\mathrm{n}=15$; fold change $=0.79$, $\mathrm{p}=0.021$ ). Moreover, in the hypertrophic/normotrophic OA stratum, the upregulation of $S T T 3 B$ expression in OA-affected versus intact cartilage appeared to be mitigated among individuals heterozygous for allele $G$ of rs6766414 $(n=6)$, yet remained significantly upregulated among homozygous carriers $(n=14)$ of allele $\mathrm{T}$ (fold change $=1.4, \mathrm{p}=2.8 \times 10^{-4}$, figure 3 ).

\section{DISCUSSION}

We applied clinical radiographic endophenotypes to stratify genome-wide association analysis of hip OA and identify six putative associations $\left(\mathrm{p}<5 \times 10^{-6}\right)$. For the strongest signals in both endophenotypes we observe consistent effects across complementary strata both in the primary analysis and against population-based controls in the secondary analyses. These signals were all fully attenuated in the non-stratified analyses against the population-based controls, consistent with our hypothesis that this stratification approach increases the sensitivity of signal detection.

rs754106, which showed the strongest association with pattern of maxJSN, resides in intron 1 of the $L R C H 1 / C H D C 1$ gene whose function has not been well characterised. However, replicating associations with knee $\mathrm{OA}$ at variants spanning intron 1 of $L R C H 1$ have been identified in samples of European descent. ${ }^{42}$ rs6766414, which showed the strongest association with bone remodelling response, is located between $S T T 3 B$ and GADL1. STT3B mediates both co-translational and posttranslational N-glycosylation of proteins and GO terms associated with this gene include glycoprotein catabolic process. ${ }^{43}$ $S T T 3 B$ encodes a catalytic subunit of the N-oligosaccaryl transferase complex, ${ }^{44}$ suggesting that altered glycosylation in OA-affected cartilage mitigates protein folding and affects intermolecular interactions. ${ }^{45}$ Thus we hypothesise that the upregulation of $S T T 3 B$ is a beneficial response among hypertrophic cases.

This study also has limitations. While the interobserver agreement of the radiographic classifiers supports the approach taken, differences in calling between observers does impact on the overall sensitivity of both the genetic association and expression analyses. Although the identified loci did not meet the Bonferroni-adjusted genome-wide significance threshold of $\mathrm{p}<5 \times 10^{-9}$ used because of the multiple discovery association analyses conducted, the putative association of variants with specific endophenotypes corroborated by substantial or full attenuation in other strata warrants replication and further functional investigation.

The hypothesis that stratification of cases into distinct endophenotypic categories provides a more direct link to the genetic basis of OA has not been previously tested systematically. However, this approach has been successfully applied to better understand disease biology in related complex traits, including joint shape and pain. ${ }^{46-50}$ The results of our analyses show that, if further replication is targeted to the specific OA endophenotypes, study power may improve from nearly $0 \%$ in the traditional approach to $80 \%$ in a sample size of $<5000$ cases. We used post hoc power calculations, applying the observed variances from the study population of interest to exemplify the difference in the statistical power of the tests we performed. Such calculations have limitations, but are useful in designing follow-up studies and in conducting meta-analyses. We address one limitation in interpreting post hoc power calculations, ascertainment bias, by examining the actual summary statistics of the previously established genome-wide significant locus (DOT1L) associated with JSN. We find that the index SNP is more strongly associated in hip OA cases with superior JSN than in the non-stratified hip OA versus controls analyses $\left(p=8 \times 10^{-6}\right.$ and $p=3 \times 10^{-4}$, respectively). As more precise phenotype definitions are accrued in the field and future replication is targeted to the specific endophenotype, the strength of the observed associations may increase and the power burden of multiple testing may be resolved. Our findings demonstrate that stratification of OA cases into more homogeneous endophenotypes can identify genes of potential functional importance otherwise obscured by disease heterogeneity. 


\section{Author affiliations}

${ }^{1}$ Department of Human Genetics, Wellcome Trust Sanger Institute, Hinxton, UK ${ }^{2}$ Department of Oncology and Metabolism, University of Sheffield, Sheffield, UK ${ }^{3} 5$ th Psychiatric Department, Dromokaiteio Psychiatric Hospital of Athens, Athens, Greece

${ }^{4}$ Department of Genomics and Computational Biology, Biogen Idec, Cambridge, Massachusetts, USA

${ }^{5}$ Department of Molecular Epidemiology, LUMC, Leiden, The Netherlands

${ }^{6}$ Department of Orthopaedics, LUMC, Leiden, The Netherlands

${ }^{7}$ Wellcome Trust Centre for Human Genetics, Oxford, UK

${ }^{8}$ Oxford Centre for Diabetes, Endocrinology and Metabolism, University of Oxford, Oxford, UK

${ }^{9}$ Academic Rheumatology, University of Nottingham, Nottingham, UK

Acknowledgements The authors thank all RAAK Study participants. The authors also thank Lucy Matthews, Robert Andrews, Siobhan Whitehead and their staff at the Wellcome Trust Sanger Institute.

Collaborators Members of the arcOGEN Consortium: N Arden, F Birrell, A Carr, K Chapman, P Deloukas, M Doherty, J Loughlin, A McCaskie, WER Ollier, A Rai, SH Ralston, TD Spector, AM Valdes, GA Wallis, JM Wilkinson and E Zeggini.

Contributors Study concept and design: IM, JMW and EZeg. Acquisition of data: All. Analysis and interpretation of data: All. Statistical analysis: KP, ST, IM, JMW and EZeg. Drafting of manuscript: IM, KP, ST and JMW. Critical revision of manuscript for important intellectual content: All. IM, JMW and EZeg had full access to all the data in the study and take responsibility for the integrity of the data and the accuracy of the data analysis.

Funding This work was supported by Arthritis Research UK (grant 19542, KP and EZ; grant 20308, KP), the Wellcome Trust (grant 098051, KP and EZ), the Joint Action Trust (grant GA1125, British Orthopaedic Association, ST and JMW) and the National Institute for Health Research funded Sheffield Bone Biomedical Research Unit (ST and JMW). This study used genotype data from arcOGEN (http://www. arcogen.org.uk/) funded by a special purpose grant from Arthritis Research UK (grant 18030). This study makes use of data generated by the Wellcome Trust Case-Control Consortium (the 1958 British Birth Cohort collection and the UK Blood Services Collection). A full list of the investigators who contributed to the generation of the data is available from http://www.wtccc.org.uk and funding for the project was provided by the Wellcome Trust under awards 076113,085475 and 090355 The Leiden University Medical Center supports the RAAK Study, while research leading to these results has received funding from the Dutch Arthritis Association (DAA 2010_017) and the IDEAL project (European Union's Seventh Framework Programme (FP7/2007-2011) under grant agreement no. 259679).

\section{Competing interests None.}

Ethics approval National Research Ethics Service.

Provenance and peer review Not commissioned; externally peer reviewed.

Open Access This is an Open Access article distributed in accordance with the terms of the Creative Commons Attribution (CC BY 4.0) license, which permits others to distribute, remix, adapt and build upon this work, for commercial use, provided the original work is properly cited. See: http://creativecommons.org/licenses/ by/4.0/

\section{REFERENCES}

1 Glyn-Jones S, Palmer AJ, Agricola R, et al. Osteoarthritis. Lancet 2015;386:376-87.

2 Hiligsmann $\mathrm{M}$, Cooper $\mathrm{C}$, Arden N, et al. Health economics in the field of osteoarthritis: an expert's consensus paper from the European Society for Clinical and Economic Aspects of Osteoporosis and Osteoarthritis (ESCEO). Semin Arthritis Rheum 2013;43:303-13.

3 Spector TD, MacGregor AJ. Risk factors for osteoarthritis: genetics. Osteoarthritis Cartilage 2004;12 (A):S39-44.

4 MacGregor AJ, Li Q, Spector TD, et al. The genetic influence on radiographic osteoarthritis is site specific at the hand, hip and knee. Rheumatology (Oxford) 2009:48:277-80

5 Valdes AM, Spector TD. Genetic epidemiology of hip and knee osteoarthritis. Nat Rev Rheumatol 2011;7:23-32.

6 Miyamoto $Y$, Mabuchi A, Shi D, et al. A functional polymorphism in the $5^{\prime}$ UTR of GDF5 is associated with susceptibility to osteoarthritis. Nat Genet 2007;39:529-33.

7 Evangelou E, Chapman K, Meulenbelt I, et al. Large-scale analysis of association between GDF5 and FRZB variants and osteoarthritis of the hip, knee, and hand. Arthritis Rheum 2009:60:1710-21.

8 Valdes AM, Evangelou E, Kerkhof HJ, et al. The GDF5 rs 143383 polymorphism is associated with osteoarthritis of the knee with genome-wide statistical significance. Ann Rheum Dis 2011;70:873-5.

9 Miyamoto Y, Shi D, Nakajima M, et al. Common variants in DVWA on chromosome 3p24.3 are associated with susceptibility to knee osteoarthritis. Nat Genet 2008:40:994-8.
10 Nakajima M, Takahashi A, Kou I, et al. New sequence variants in HLA class II/III region associated with susceptibility to knee osteoarthritis identified by genome-wide association study. PLOS ONE 2010;5:e9723.

11 Kerkhof HJ, Lories RJ, Meulenbelt I, et al. A genome-wide association study identifies an osteoarthritis susceptibility locus on chromosome 7q22. Arthritis Rheum 2010:62:499-510.

12 Evangelou E, Valdes AM, Kerkhof $\mathrm{HJ}$, et al. Meta-analysis of genome-wide association studies confirms a susceptibility locus for knee osteoarthritis on chromosome 7q22. Ann Rheum Dis 2011;70:349-55.

13 Day-Williams AG, Southam L, Panoutsopoulou K, et al. A variant in MCF2L is associated with osteoarthritis. Am J Hum Genet 2011;89:446-50.

14 Zeggini E, Panoutsopoulou K, Southam L, et al., arcOGEN Consortium; arcOGEN Collaborators. Identification of new susceptibility loci for osteoarthritis (arcOGEN): a genome-wide association study. Lancet 2012;380:815-23.

15 Evangelou E, Valdes AM, Castano-Betancourt MC, et al. The DOT1L rs12982744 polymorphism is associated with osteoarthritis of the hip with genome-wide statistical significance in males. Ann Rheum Dis 2013:72:1264-5.

16 Evangelou E, Kerkhof HJ, Styrkarsdottir U, et al. A meta-analysis of genome-wide association studies identifies novel variants associated with osteoarthritis of the hip. Ann Rheum Dis 2014;73:2130-6.

17 Styrkarsdottir U, Thorleifsson G, Helgadottir HT, et al. Severe osteoarthritis of the hand associates with common variants within the ALDH1A2 gene and with rare variants at 1p31. Nat Genet 2014;46:498-502.

18 Zengini E, Finan C, Wilkinson JM. The Genetic Epidemiological Landscape of Hip and Knee Osteoarthritis: Where Are We Now and Where Are We Going? J Rheumatol 2016;43:260-6.

19 Kerkhof HJ, Meulenbelt I, Akune T, et al. Recommendations for standardization and phenotype definitions in genetic studies of osteoarthritis: the TREAT-OA consortium. Osteoarthritis Cartilage 2011;19:254-64

20 Castano Betancourt MC, Cailotto F, Kerkhof HJ, et al. Genome-wide association and functional studies identify the DOT1L gene to be involved in cartilage thickness and hip osteoarthritis. Proc Natl Acad Sci USA 2012;109: 8218-23.

21 Kellgren JH, Lawrence JS. Radiological assessment of osteo-arthrosis. Ann Rheum Dis 1957:16:494-502.

22 Altman RD, Gold GE. Atlas of individual radiographic features in osteoarthritis, revised. Osteoarthritis Cartilage 2007:15 A):A1-56.

23 Bombelli R. Osteoarthritis of the hip: classification and pathogenesis. 2nd edn. Berlin: Springer-Verlag, 1983.

24 Resnick D. Patterns of migration of the femoral head in osteoarthritis of the hip Roentgenographic-pathologic correlation and comparison with rheumatoid arthritis. Am I Roentgenol Radium Ther Nucl Med 1975;124:62-74.

25 Altman R, Alarcón G, Appelrouth D, et al. The American College of Rheumatology criteria for the classification and reporting of osteoarthritis of the hip. Arthritis Rheum 1991;34:505-14.

26 Ledingham J, Dawson S, Preston B, et al. Radiographic patterns and associations of osteoarthritis of the hip. Ann Rheum Dis 1992;51:1111-16.

27 Lanyon $\mathrm{P}$, Muir K, Doherty $\mathrm{S}$, et al. Influence of radiographic phenotype on risk of hip osteoarthritis within families. Ann Rheum Dis 2004;63:259-63.

28 Ledingham J, Dawson S, Preston B, et al. Radiographic progression of hospital referred osteoarthritis of the hip. Ann Rheum Dis 1993;52:263-7.

29 Dougados M, Gueguen A, Nguyen M, et al. Radiological progression of hip osteoarthritis: definition, risk factors and correlations with clinical status. Ann Rheum Dis 1996;55:356-62.

30 Bissacotti JF, Ritter MA, Faris PM, et al. A new radiographic evaluation of primary osteoarthritis. Orthopedics 1994;17:927-30.

31 Schnitzler CM, Mesquita JM, Wane L. Bone histomorphometry of the iliac crest, and spinal fracture prevalence in atrophic and hypertrophic osteoarthritis of the hip. Osteoporos Int 1992:2:186-94.

32 Castaño-Betancourt MC, Rivadeneira F, Bierma-Zeinstra S, et al. Bone parameters across different types of hip osteoarthritis and their relationship to osteoporotic fracture risk. Arthritis Rheum 2013:65:693-700.

33 Bierma-Zeinstra SM, Koes BW. Risk factors and prognostic factors of hip and knee osteoarthritis. Nat Clin Pract Rheumatol 2007:3:78-85.

34 Landis JR, Koch GG. The measurement of observer agreement for categorical data. Biometrics 1977:33:159-74.

35 Genomes Project $C$, Abecasis GR, Altshuler D, et al. A map of human genome variation from population-scale sequencing. Nature 2010;467:1061-73.

36 Marchini J, Howie B, Myers $\mathrm{S}$, et al. A new multipoint method for genome-wide association studies by imputation of genotypes. Nat Genet 2007;39:906-13.

$37 \mathrm{Ma}$ C, Blackwell T, Boehnke M, et al. Recommended joint and meta-analysis strategies for case-control association testing of single low-count variants. Genet Epidemiol 2013:37:539-50.

38 Mägi R, Morris AP. GWAMA: software for genome-wide association meta-analysis. BMC Bioinformatics 2010;11:288.

39 Gauderman WM, J M. (cited 08 June 2015).

40 Ramos YF, den Hollander W, Bovée JV, et al. Genes involved in the osteoarthritis process identified through genome wide expression analysis in articular cartilage; 


\section{Clinical and epidemiological research}

the RAAK study. PLOS ONE 2014 Jul 23;9(7):e103056. doi:10.1371/journal.pone. 0103056

41 Goeman JJ, van de Geer SA, de Kort F, et al. A global test for groups of genes: testing association with a clinical outcome. Bioinformatics 2004:20:93-9.

42 Spector TD, Reneland RH, Mah S, et al. Association between a variation in LRCH1 and knee osteoarthritis: a genome-wide single-nucleotide polymorphism association study using DNA pooling. Arthritis Rheum 2006;54:524-32.

43 Shrimal S, Cherepanova NA, Gilmore R. Cotranslational and posttranslocational $\mathrm{N}$-glycosylation of proteins in the endoplasmic reticulum. Semin Cell Dev Biol 2015:41:71-8.

44 Breitling J, Aebi M. N-linked protein glycosylation in the endoplasmic reticulum. Cold Spring Harb Perspect Biol 2013;5:a013359.

45 Zaia J, Boynton RE, McIntosh A, et al. Post-translational modifications in cartilage oligomeric matrix protein. Characterization of the N-linked oligosaccharides by matrix-assisted laser desorption ionization time-of-flight mass spectrometry. J Biol Chem 1997;272:14120-6.
46 Agricola R, Leyland KM, Bierma-Zeinstra SM, et al. Validation of statistical shape modelling to predict hip osteoarthritis in females: data from two prospective cohort studies (Cohort Hip and Cohort Knee and Chingford). Rheumatology (Oxford) 2015:54:2033-41.

47 Waarsing JH, Kloppenburg M, Slagboom PE, et al. Osteoarthritis susceptibility genes influence the association between hip morphology and osteoarthritis. Arthritis Rheum 2011;63:1349-54.

48 Lindner C, Thiagarajah S, Wilkinson JM, et al., arcOGEN Consortium. Investigation of association between hip osteoarthritis susceptibility loci and radiographic proximal femur shape. Arthritis Rheum 2015;67: 2076-84.

49 Baker-Lepain JC, Lynch JA, Parimi N, et al. Variant alleles of the Wnt antagonist FRZB are determinants of hip shape and modify the relationship between hip shape and osteoarthritis. Arthritis Rheum 2012;64:1457-65.

50 Tracey I. Can neuroimaging studies identify pain endophenotypes in humans? Nat Rev Neurol 2011;7:173-81. 